Case Report

\title{
GeneXpert's Performance in the Diagnosis of Pulmonary and Extrapulmonary Tuberculosis
}

\author{
Mouhib Hanane, Zahir Hanane, Arsalane Lamiae, Kamouni Youssef, Miloudi Mouhcine, \\ Zouhair Said
}

Laboratory of Bacteriology-virology and Molecular Biology Military Hospital, Mohammed VI UHC, Marrakech, Morocco

Email address:

mouhib.hanane1@gmail.com (M. Hanane)

\section{To cite this article:}

Mouhib Hanane, Zahir Hanane, Arsalane Lamiae, Kamouni Youssef, Miloudi Mouhcine, Zouhair Said. GeneXpert's Performance in the Diagnosis of Pulmonary and Extrapulmonary Tuberculosis. American Journal of Laboratory Medicine. Vol. 5, No. 1, 2020 , pp. $14-17$. doi: $10.11648 /$ j.ajlm.20200501.12

Received: November 13, 2019; Accepted: December 4, 2019; Published: January 7, 2020

\begin{abstract}
The diagnosis of tuberculosis is based on the isolation of acid-fast bacilli (AFB) on direct examination or on the culture isolation of mycobacteria tuberculosis complex (MCT). Currently, the introduction of molecular biology, essentially the Xpert / MTB / Rif or GeneXpertR test has increased the sensitivity and speed of diagnosis. The objective of our study is the evaluation of the performance of the Xpert / MTB / Rif molecular technique in the diagnosis of pulmonary and extrapulmonary tuberculosis. This is a descriptive retrospective study over a period of 22 months (June 2016 - April 2018). The study concerned all pulmonary and extrapulmonary specimens sent to the laboratory of bacteriology-virology and molecular biology of the HMA Marrakech for suspicion of tuberculosis. Diagnosis was performed on all specimens by direct examination on Ziehl-Neelsen stained smears, culture on Loweinstein Jensen solid medium (LJ) and GeneXpert molecular technical. Our study included 305 patients, $69 \%$ were men and $31 \%$ women. The clinical samples included in the study consisted of different specimens with a prevalenceof sputum $(\mathrm{n}=127)$. Positivity was higher for GeneXpert MTB / RIF than for Ziehl-Neelsen and culture with respectively $18 \%(\mathrm{n}=54)$ for GeneXpert against $13 \%(\mathrm{n}=40)$ for culture and $8 \%(\mathrm{n}=24)$ for Ziehl-Neelsen. On all positive direct examination specimens, GeneXpert was positive in $100 \%$ of cases, on negative direct examination samples, GeneXpert was positive in $5 \%$ of cases. The positivity rate of GeneXpert was $10.6 \%$ for samples with negative culture and $100 \%$ for positive culture samples. The performance of GeneXpert was variable depending on the nature of the samples: sputum (23\%), biopsy $(15 \%)$, pleural fluid $(13 \%)$. Four cases of resistance to rifampicin have been detected. Our study shows the great contribution of GeneXpert for the diagnosis of pulmonary and extrapulmonary tuberculosis as well as its place in the determination of multidrug resistance.
\end{abstract}

Keywords: Tuberculosis, GeneXpert, Culture, Direct Examination

\section{Introduction}

Tuberculosis is a real public health problem and is the leading infectious disease in the world with an overall incidence according to WHO of 10.4 million cases in 2016 [1]. It is estimated that nearly 2 billion people, or one third of the world's population, is the reservoir of bacilli [2]. Tuberculosis is responsible for 1.5 million deaths worldwide, $95 \%$ of which are in low- and middle-income countries [3]. In Morocco 27,745 new cases were recorded with an incidence of 82 new cases per 100,000 inhabitants and a predominance of extrapulmonary tuberculosis [4]. The diagnosis of tuberculosis is based on the isolation of acid-fast bacilli (AFB) on direct examination or on isolation of mycobacteria tuberculosis complex (MCT) in culture. However, the detection of Mycobacterium tuberculosis complex in extrapulmonary locations remains difficult because of the presence of mycobacteria in small clinical samples compared to pulmonary infections [5]. Currently, the establishment of molecular biology, essentially the Xpert / MTB / Rif or GeneXpertR test that was approved by the WHO in December 2010 , for the first-line diagnosis of pulmonary tuberculosis in 
patients living with HIV or suspected of developing multidrug-resistant tuberculosis [6], this tool has increased the sensitivity and speed of diagnosis. However, the limit of PCR is its excessive cost. The objective of our study is the evaluation of the performance of the Xpert / MTB / Rif molecular technique in the diagnosis of pulmonary and extrapulmonary tuberculosis.

\section{Materials and Methods}

This is a retrospective descriptive and analytical study consisting of a collection from the laboratory of bacteriology-virology and molecular biology of the HMA Marrakech, the simultaneous results of Ziehl-Neelsen and GeneXpert MTB / RIF as well as the culture of 305 samples sent to our training for suspicion of tuberculosis. The study was carried out over a 22-month period from June 2016 to
April 2018. The diagnosis was made on all samples by direct examination on Ziehl-Neelsen stained smears, by culture on the solid medium of Loweinstein Jensen (LJ) and the molecular technique of GeneXpert. The study of rifampicin resistance was confirmed by GeneXpert MTB / RIF. The statistical data of our study were entered with the Microsoft Excel 2007 calculation software.

\section{Results}

Our study included 305 patients, 69\% were men and 31\% were women. The samples received came from different departments [Figure 1]. The clinical samples included in the study consisted mainly of sputum $(n=127)$, pleural fluid biopsies $(n=51)(n=39)$, ascites fluid $(n=21)$, pus $(n=21),=$ $18)$, bronchial aspiration $(\mathrm{n}=13)$ and $\operatorname{CSF}(\mathrm{n}=10)$ [Figure 2].

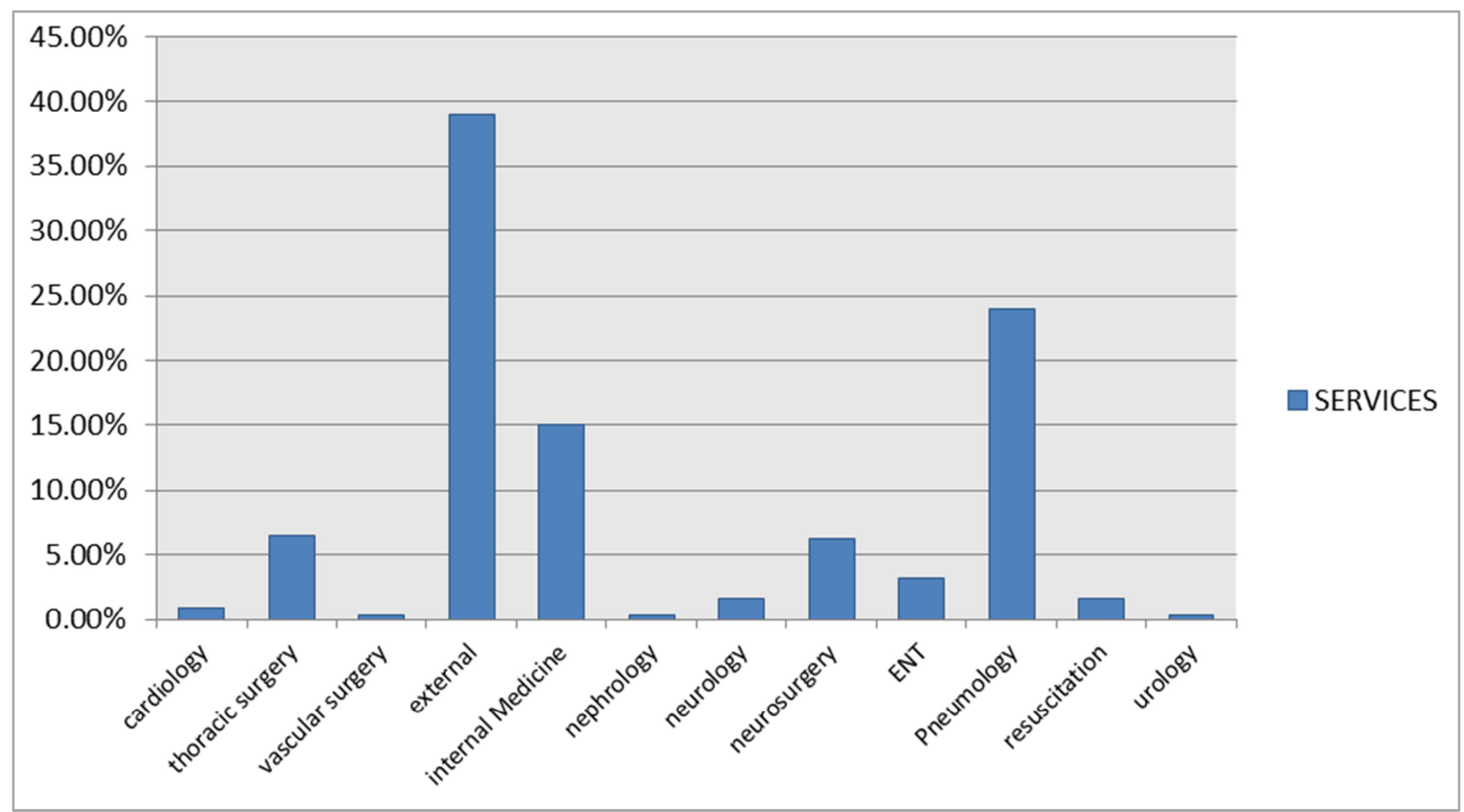

Figure 1. Distribution of levies by services.

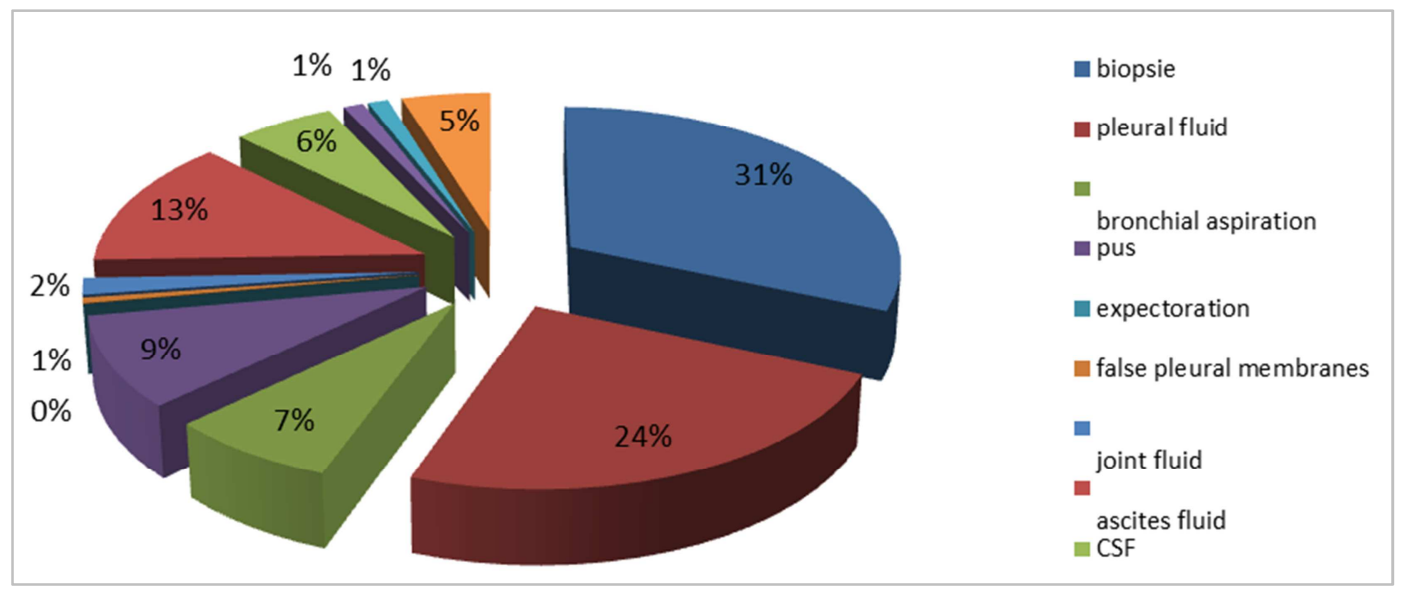

Figure 2. The clinical samples included in the study. 
GeneXpert against 13\% $(\mathrm{n}=40)$ for culture and $8 \%(\mathrm{n}=24)$ for Ziehl-Neelsen [Figure 3]. The sensitivity and total specificity of GeneXpert was respectively $100 \%$ and $89 \%$. On all positive direct examination specimens, GeneXpert was positive in $100 \%$ of cases, on negative direct examination samples, GeneXpert was positive in $5 \%$ of cases. The positivity rate of GeneXpert was $10.6 \%$ for culture-negative samples and $100 \%$ for culture-positive samples. The positivity rate of GeneXpert was variable according to the nature of the samples: in the sputum $(23 \%)$, in the biopsies $(15 \%)$, in the pleural fluid (13\%), however the sensitivity of GeneXpert for LCR, pleural fluid, Bronchial suction fluid, joint fluid and ascites fluid is $100 \%$.

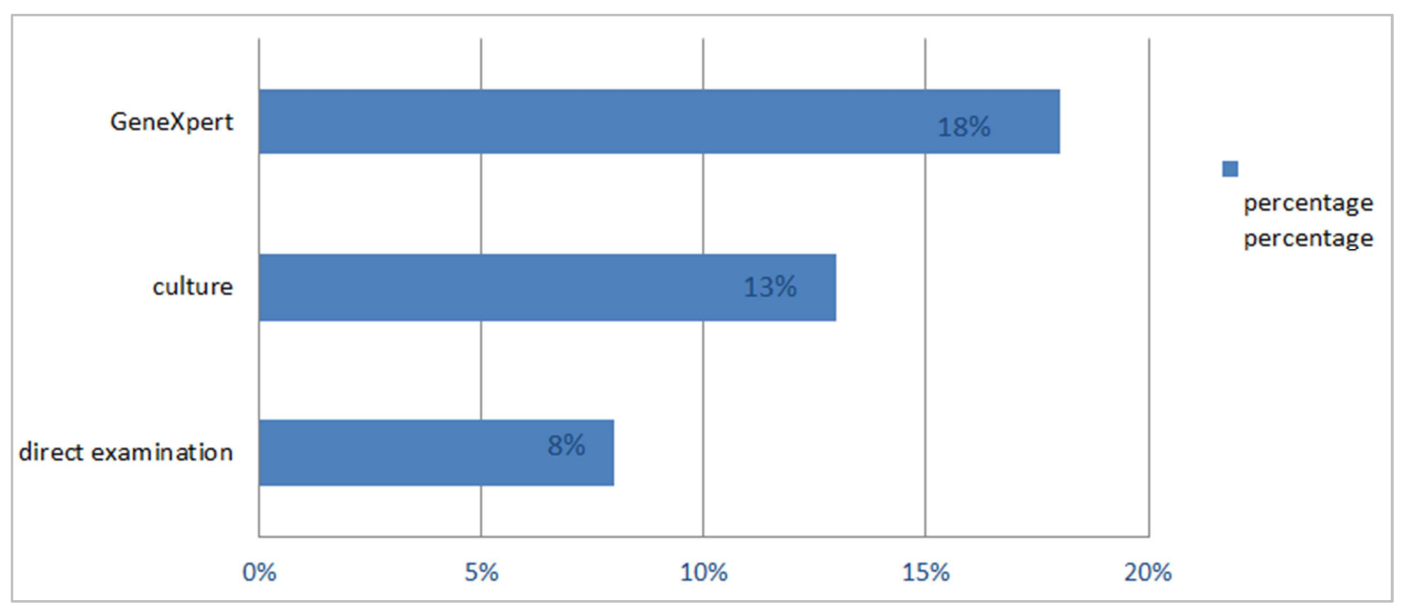

Figure 3. Positivity rate of GeneXpert compared to culture and direct examination.

Our study included 180 respiratory specimens including bronchial aspiration fluid, pleural fluid, protected distal specimens and sputum, of which $8 \%(n=14)$ had a positive culture, $3 \%(\mathrm{n}=5)$ had a contaminated, while culture was sterile in $89.4 \%$ of cases $(n=161)$.

However GeneXpert was positive in $20 \%$ of the cases $(n=$ 36), distributed as follows:

- $38 \%$ of cases $(n=14)$ had a positive culture.

- $55 \%$ of cases $(n=20)$ had a sterile culture.

- $5 \%$ of cases $(n=2)$ had a contaminated culture.

During the period of this study, we received 125 extra-respiratory specimens. The culture was positive in $3.2 \%$ of cases $(n=4)$, sterile in $88 \%(n=111)$ and contaminated in $3.2 \%(n=4)$. GeneXpert was positive in $14.4 \%$ of cases $(n=$ 18), 10 of which had a negative culture.

Four cases of resistance to rifampicin were detected at a frequency of $7 \%$.

\section{Discussion}

Tuberculosis is a contagious disease that comes under two nosological entities: latent tuberculosis infection and tuberculosis disease. The evolution towards tuberculosis disease is due to a certain number of factors, such as the risky medical acts (intubation, extubation, bronchial drainage by physiotherapy, necropsy), also the age less than 2 years, the immunosuppressive, the hematological malignancies, carcinoma of the head suddenly, type 2 diabetes, silicosis, weight loss, alcoholism is renal failure [7]. The diagnosis of tuberculosis is based on clinical and radiological arguments, but confirmation is necessarily bacteriological and / or histological. The culture remains the gold standard. Technological progress, especially in the field of molecular biology, is providing the clinician with new means of diagnosing tuberculosis. The Xpert ${ }^{\circledR}$ MTB / RIF test has proved its usefulness in the diagnosis of tuberculosis [8], it is an automated real-time PCR that detects the presence of $\mathrm{Mt}$ along with the most frequent mutations (resistance rifampicin) in less than 2 hours [2]. The performance of GeneXpert in the diagnosis of pulmonary and extra-pulmonary tuberculosis is variable according to the studies [9]. In our study GeneXpert has a much higher sensitivity and specificity than Ziehl-Neelsen and culture. Our results are consistent with other studies that have confirmed the efficacy of GeneXpert in the diagnosis of pulmonary and extra-pulmonary tuberculosis [10].

On all positive direct examination specimens, GeneXpert was positive in $100 \%$ of cases, in direct negative specimens GeneXpert was positive in 5\% of cases. This can be explained by the fact that the extraction of nucleic acids is done automatically in the cartridge, there is neither loss nor contamination by DNA [11]. The positivity rate of GeneXpert was $10.6 \%$ for culture-negative samples and $100 \%$ for culture-positive samples. These values are comparable to those obtained by other studies [12].

The performance of GeneXpert and culture over microscopy can be explained by the fact that the detection limits of culture and GeneXpert would be 10-100 CFU / ml and $131 \mathrm{CFU} / \mathrm{ml}$ respectively, whereas microscopy would be between 5,000 and 10,000 CFU / $\mathrm{ml}$. [13]

GeneXpert's evaluation of the results and in comparison with the results of the culture, GeneXpert revealed a higher rate of positivity.

This makes GeneXpert a powerful tool for fast detection (about 2 hours) for MTB. An additional benefit of the GeneXpert MTB / RIF test is the modality of sample 
processing when treated samples can be analyzed directly. [14]

In our study, the sensitivity of GeneXpert for CSF, pleural fluid, bronchial aspiration fluid, joint fluid and ascites fluid is $100 \%$. Indeed, numerous studies have been able to show high sensitivity levels of GeneXpert in extra-pulmonary samples $[12,5]$. These results confirm the good performance of GX in the diagnosis of TBEP despite the pauci bacillary nature of extrapulmonary clinical specimens.

The use of GeneXpert MTB / RIF detected a 7\% resistance rate to Rifampicin, a marker for the prediction of multi-resistance for tuberculosis, this frequency remains lower than that found in other studies as well as to the WHO estimate of $10 \%$ [15].

A study conducted in Iran of the contribution of GeneXpert in the detection of rifampicin resistance has shown that GeneXpert can be a rapid and useful method for the diagnosis of rifampicin resistance. In addition, GeneXpert can provide results in a short time, as it is not necessary to wait for smear results as in the conventional method. As a result, the treatment can be started more quickly [16].

\section{Conclusion}

Our study shows the great contribution of GeneXpert for the diagnosis of tuberculosis and its place in the determination of multidrug resistance, allowing both a rapid diagnosis of tuberculosis and a good sensitivity and specificity. Thus the GeneXpert MTB / RIF test can be used as a confirmatory test for the detection of tuberculosis.

\section{References}

[1] Global tuberculosis report 2017. World Health Organization; 2017.

[2] Jabri. H, Lakhdar. N, ElKhattabi. W, Afif. H. Les moyens diagnostiques de la tuberculose. Rev PneumolClin2016; 72: 320-325.

[3] World Health Organization. (2015). Global tuberculosis report 2015, 20th ed. World Health Organization.

[4] Direction de l'épidémiologie et de la lutte contre les maladies. Maroc: Ministère de la santé publique; 2014.

[5] Hillemann D, Rusch-Gerdes S, Boehme C, Richter E. Rapid molecular detection of extrapulmonary tuberculosis by the automated GeneXpert MTB/RIF system. J Clin Microbiol 2011; 49 (4): 1202-5.
[6] World Health Organization. Policy Statement: Automated Real-Time Nucleic Acid Amplification Technology for Rapid and Simultaneous Detection of Tuberculosis and Rifampicin Resistance: Xpert MTB/RIF system. Geneva, Switzerland: World Health Organization; 2015.

[7] Erkens CG, Kamphorst M, Abubakar I, et al. Tuberculosis contact investigation in low prevalence countries: a European consensus. EurRespir J 2010; 36: 925- 49.

[8] Horoa. K, Guessanb. R, Koffia. M, Kouamé-N’Takpéc. N et all. TestXpert ${ }^{\circledR}$ MTB/RIF et dépistage des nouveaux cas de tuberculose pulmonaire en routine dans une zone de haute endémicité tuberculeuse. Rev Mal Respir 2017; 34: 749—757

[9] Diallo. A, Issifi Kollo. A, Camara. M et all. Performance du GeneXpert MTB/RIF® dans le diagnostic de la tuberculose extra-pulmonaire à Dakar. PAMJ 2010-2015.

[10] Tortoli E, Russo C, Piersimoni C, Mazzola E, Dal Monte P, Pascarella M, et al. Clinical validation of Xpert MTB/RIF for the diagnosis of extrapulmonary tuberculosis. Eur Respir J 2012; 40 (2): 442-7.

[11] Slim-Saidi L, Mehiri-Zeghal E, Ghariani A, Tritar F. Nouvelles méthodes de diagnostic de la tuberculose. Rev PneumolClin 2015; 71: 110-21.

[12] Vadwai V, Boehme C, Nabeta P, Shetty A, Alland D, Rodrigues C. Xpert MTB/RIF: a new pillar in diagnosis of extrapulmonary tuberculosis? JClinMicrobiol2011; 49 (7): 2540-5.

[13] Marlowe EM, Novak-Weekley SM, Cumpio J, Sharp SE, Momeny MA, Babst A, et all. Evaluation of the Cepheid Xpert MTB/RIF assay for direct detection of Mycobacterium tuberculosis complex in respiratory specimens. J Clin Microbiol2011; 49 (4): 1621-3.

[14] Lupande D, Kaishusha D, Mihigo C et all. GeneXpert MTB/RIF dans le dépistage de la tuberculose pulmonaire à l'Hôpital Provincial Général de Référence de Bukavu, à l'Est de la République Démocratique du Congo: quelles leçons tirées après 10 mois d'utilisation?PAMJ 2017; 27: 260.

[15] Pandey S, Congdon J, Mcinnes B, Pop A et all. Evaluation of the GeneXpert MTB/RIF assay on extrapulmonary and respiratory samples other than sputum: a low burden country experience. Pathology 2017; 49 (1): 70-74.

[16] Williamson DA, Basu I, Bower J, Freeman JT, Henderson G, Roberts SA. An evaluation of the Xpert MTB/RIF assay and detection of false-positive rifampicin resistance in Mycobacterium tuberculosis. DiagnMicrobiol Infect Dis 2012; 74 (2): 207-9. 